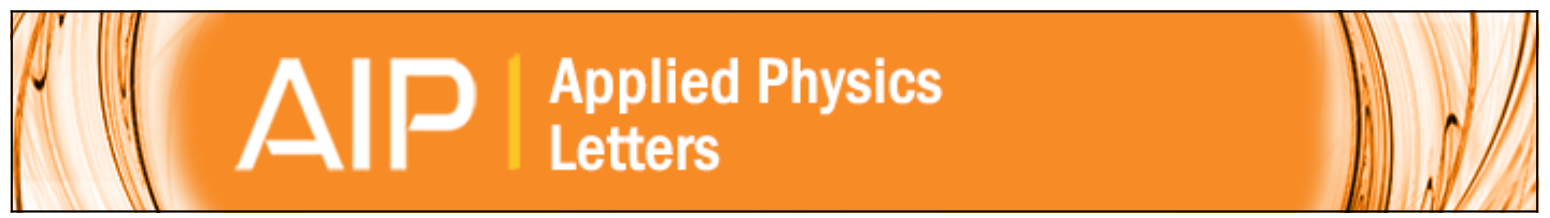

\title{
Reduced frequency noise in superconducting resonators
}

R. Barends, N. Vercruyssen, A. Endo, P. J. de Visser, T. Zijlstra, T. M. Klapwijk, and J. J. A. Baselmans

Citation: Applied Physics Letters 97, 033507 (2010); doi: 10.1063/1.3467052

View online: http://dx.doi.org/10.1063/1.3467052

View Table of Contents: http://scitation.aip.org/content/aip/journal/apl/97/3?ver=pdfcov

Published by the AIP Publishing

\section{Articles you may be interested in}

Frequency-tunable superconducting resonators via nonlinear kinetic inductance Appl. Phys. Lett. 107, 062601 (2015); 10.1063/1.4927444

Reducing intrinsic loss in superconducting resonators by surface treatment and deep etching of silicon substrates

Appl. Phys. Lett. 106, 182601 (2015); 10.1063/1.4919761

Anomalous response of superconducting titanium nitride resonators to terahertz radiation Appl. Phys. Lett. 105, 192601 (2014); 10.1063/1.4901536

Etch induced microwave losses in titanium nitride superconducting resonators Appl. Phys. Lett. 100, 262605 (2012); 10.1063/1.4729623

Contribution of dielectrics to frequency and noise of $\mathrm{NbTiN}$ superconducting resonators Appl. Phys. Lett. 92, 223502 (2008); 10.1063/1.2937837

\section{Precise temperature control for cryogenic research}

\section{Model 372}

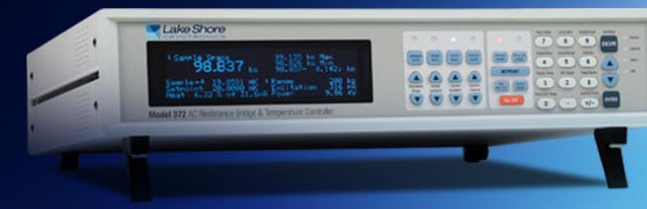




\title{
Reduced frequency noise in superconducting resonators
}

\author{
R. Barends, ${ }^{1, a)}$ N. Vercruyssen, ${ }^{1}$ A. Endo, ${ }^{1}$ P. J. de Visser, ${ }^{1,2}$ T. Zijlstra, ${ }^{1}$ \\ T. M. Klapwijk, ${ }^{1}$ and J. J. A. Baselmans ${ }^{2}$ \\ ${ }^{1}$ Kavli Institute of NanoScience, Faculty of Applied Sciences, Delft University of Technology, Lorentzweg 1, \\ 2628 CJ Delft, The Netherlands \\ ${ }^{2}$ SRON Netherlands Institute for Space Research, Sorbonnelaan 2, 3584 CA Utrecht, The Netherlands
}

(Received 28 May 2010; accepted 30 June 2010; published online 23 July 2010)

\begin{abstract}
We report a reduction in the frequency noise in coplanar waveguide superconducting resonators. The reduction of $7 \mathrm{~dB}$ is achieved by removing the exposed dielectric substrate surface from the region with high electric fields and by using NbTiN. In a model-analysis the surface of NbTiN is found to be a negligible source of noise, experimentally supported by a comparison with NbTiN on $\mathrm{SiO}_{x}$ resonators. The reduction is additive to decreasing the noise by widening the resonators. (C) 2010 American Institute of Physics. [doi:10.1063/1.3467052]
\end{abstract}

The development of large and sensitive imaging arrays for far infrared astronomical instrumentation is rapidly progressing with microwave kinetic inductance detectors. ${ }^{1}$ Arrays have already been taken to ground-based telescopes, 2,3 and readout using frequency domain multiplexing has been demonstrated. ${ }^{4}$ The frequency noise in these superconducting resonators is two to three orders of magnitude above the fundamental limit of generation-recombination noise. The noise has been conjectured to arise from dipole two-level systems (TLS) in surface dielectrics, ${ }^{5}$ which is supported by recent experiments: The surface has been shown to be a dominant source of noise, by measurements on the width scaling by Gao et al. ${ }^{6}$ Moreover, we have shown that introducing dielectrics by covering the resonators with $\mathrm{SiO}_{x}$ leads to an increase in the noise. ${ }^{7}$ Noroozian $e t$ al. showed that the noise arises predominantly from the capacitive portion of the resonator by using a lumped element capacitor. ${ }^{8}$ Noise reduction can be achieved by widening the resonator, in essence decreasing the surface to volume ratio. ${ }^{6,8,9}$ However, the practically limiting source of noise remains to be identified and reduced.

Here, we show that the noise can be decreased by minimizing the dielectrics in the resonator itself. The lowest noise is achieved by using NbTiN deposited on a hydrogen passivated substrate and by removing the substrate from the region with the largest electric fields. The combination of removing the substrate and widening the resonator leads to a reduction of $9 \mathrm{~dB}$ for our first-generation resonators.

Dipole TLS are known to influence the temperature dependent permittivity $\epsilon,{ }^{10}$ and consequently the resonance frequency. The superposition of permittivity and complex conductivity $\left(\sigma_{1}-i \sigma_{2}\right)$ controls the resonance frequency ${ }^{7,11}$

$$
\frac{\delta f_{0}}{f_{0}}=\frac{\alpha \beta}{4} \frac{\delta \sigma_{2}}{\sigma_{2}}-\frac{F}{2} \frac{\delta \epsilon}{\epsilon},
$$

with $\alpha$ the kinetic inductance fraction and $\beta=1$ for the thick film and $\beta=2$ for the thin film limit. The filling factor $F$ (Ref. 11) takes into account the location of the dielectric and weighs its contribution to the frequency by the electric

\footnotetext{
${ }^{a}$ Present address: Department of Physics, University of California, Santa Barbara, California 93106, USA. Electronic mail: rbarends@physics.ucsb.edu.
}

field energy inside our resonator geometry. It is defined by $F=(1 / 2) \epsilon_{0} \epsilon_{h} \iiint_{V_{h}}|\vec{E}(\vec{r})|^{2} d \vec{r} /(1 / 4) C V_{r}^{2} l$, with $C$ the capacitance per unit length, $V_{r}$ the standing wave voltage, $l$ the length of the resonator, and $\epsilon_{h}$ the relative permittivity and $V_{h}$ the volume of the dielectric hosting the TLS.

Similarly, dipole TLS cause frequency noise through the time-varying permittivity $\epsilon(\vec{r}, t) .{ }^{5}$ Consequently, the power spectral density of the permittivity $S_{\epsilon}=2 \epsilon_{0}^{2} \mathcal{F}\left\{\left\langle\epsilon_{h}(t) \epsilon_{h}(t-\tau)\right\rangle\right\}$ translates to frequency noise, ${ }^{6}$

$$
\frac{S_{f_{0}}}{f_{0}^{2}}=\frac{\frac{1}{4} 2 \iiint_{V_{h}} S_{\epsilon}|\vec{E}(\vec{r})|^{4} d \vec{r}}{\left(\frac{1}{4} C V_{r}^{2} l\right)^{2}},
$$

with $S_{f_{0}} / f_{0}^{2}$ the normalized frequency noise.

In order to identify the contribution of the various surfaces to noise we calculate the effect of a hypothetical surface layer with thickness $t \rightarrow 0$ containing dipole TLS. The electric fields in the coplanar waveguide geometry are calculated using the potential matrix to find the charge density. The approach is detailed in Ref. 12. We adopt the assumption by Gao et $\mathrm{al}^{6}{ }^{6}$ that the noise spectral density follows: $S_{\epsilon}$ $=\epsilon_{0}^{2} \kappa / \sqrt{|E|^{2}+E_{s}^{2}}$, with $E_{s}$ the saturation field, following the saturation of microwave loss due to TLS. ${ }^{10}$
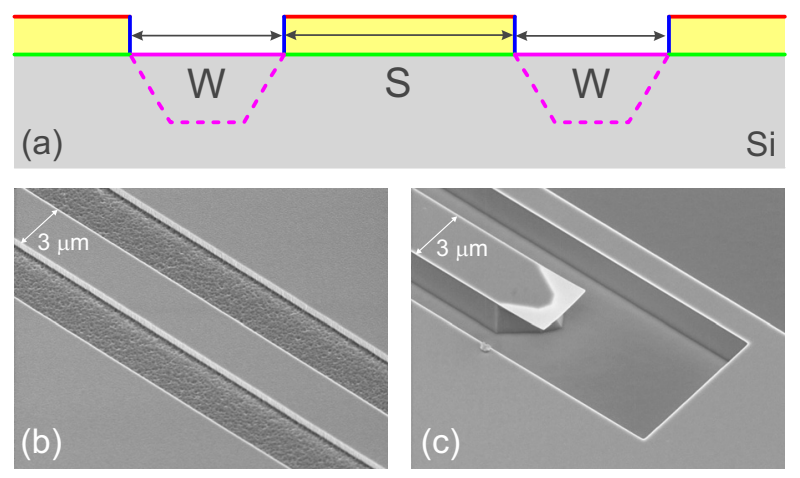

FIG. 1. (Color online) (a) The coplanar waveguide with the top metal surface, the exposed substrate, the substrate-metal interface and the etched metal edges outlined. (b) A scanning electron microscope image from the standard resonator design. (c) The etched grooves near the open end of the resonator, its cross section is outlined in (a) (dashed). The central line width is $S=3 \mu \mathrm{m}$ in both images. 


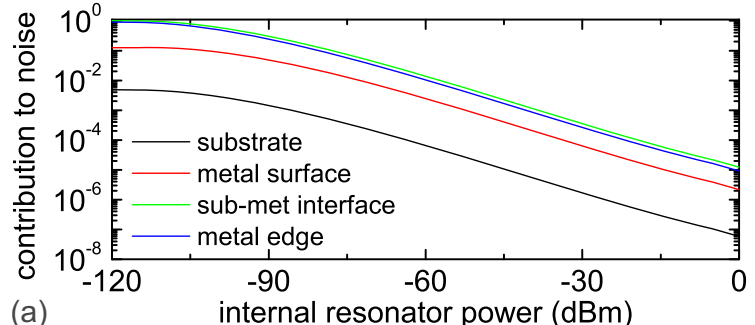

(a)
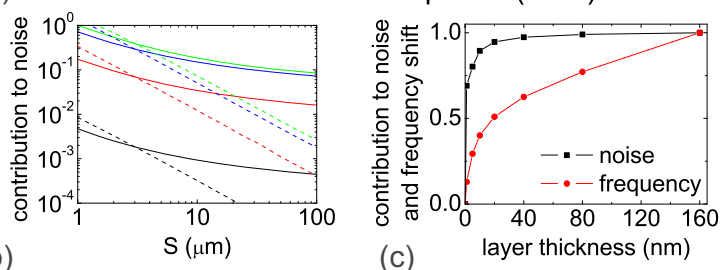

FIG. 2. (Color online) (a) The power dependence of the normalized contribution to noise using Eq. (2), for a TLS distribution placed on the exposed substrate surface, top metal surface, substrate-metal (sub-met) interface and etched metal edges $\left(E_{s}=5 \mathrm{kV} / \mathrm{m}\right.$, see Ref. 12). (b) The normalized contribution to noise of the dielectric layers vs central line width $S$, for $W=2 \mu \mathrm{m}$ (solid) and $W=2 S / 3$ (dashed). (c) The normalized contribution to noise and frequency shift for a dielectric layer with finite thickness on top of the metal. Calculations are done for $\epsilon_{h}=1$.

The hypothetical layer is placed along each of the outlined surfaces in Fig. 1(a), the exposed substrate surface, the top metal surface, the etched metal edges, and the substratemetal interface. The results are shown in Figs. 2(a) and 2(b). Importantly, the contribution to the frequency noise is about two orders of magnitude larger when the layer is placed on surfaces adjacent to the metal than when placed on the exposed substrate. This is due to the high electric fields close to the metal. Moreover, we find that the noise follows $S_{f_{0}} / f_{0}^{2}$ $\propto 1 / P_{\text {int }}^{0.5}$ in the relevant power range, with $P_{\text {int }}$ the internal resonator power. ${ }^{13}$ In addition, when widening the resonator geometry the noise decreases. We have also calculated the influence of a metal surface dielectric with finite thickness $t$ on the noise as well as the frequency shift, i.e., Eqs. (1) and (2). We find that for the noise, only the first few nanometers matter, whereas the full volume influences the frequency shift. This is consistent with our previous experiments, where we showed that frequency deviations arise from the bulk of the dielectric while noise arises predominantly at surfaces and interfaces. ${ }^{7}$ The difference arises from the surface layer being weighed by $|\vec{E}(\vec{r})|^{4}$ for the noise and $|\vec{E}(\vec{r})|^{2}$ for the frequency shift. Interestingly, the power and width dependence is very similar for each of the surfaces, and identification of the dominant noise source can be done only by removing or altering a specific surface.

In order to identify and reduce the dominant noise source, we have fabricated a series of devices aimed at addressing a specific surface, see Fig. 1. We use NbTiN quarterwave coplanar waveguide resonators ${ }^{1,7}$ with varying geometry or composition. Resonance frequencies lie between 3-5 GHz. As a reference a $300 \mathrm{~nm}$ NbTiN film is dc sputtered on a hydrogen passivated high resistivity $(>1 \mathrm{k} \Omega \mathrm{cm})\langle 100\rangle$-oriented Si wafer. Patterning is done using $\mathrm{SF}_{6} / \mathrm{O}_{2}$ reactive ion etching. The critical temperature is $T_{c}=14.8 \mathrm{~K}$, the low temperature resistivity is $\rho$ $=170 \mu \Omega \mathrm{cm}$ and the residual resistance ratio is 0.94 . To identify the importance of using hydrogen passivated $\mathrm{Si}$, a $300 \mathrm{~nm}$ NbTiN film has been deposited on the native oxide

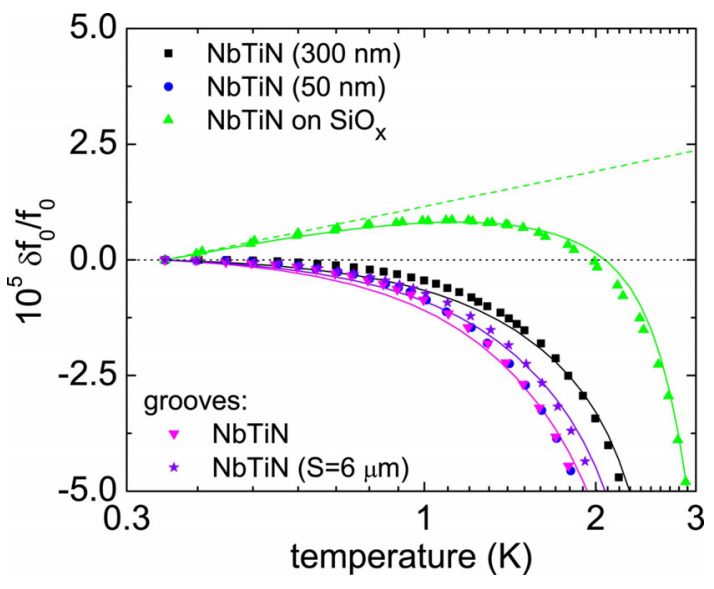

FIG. 3. (Color online) The temperature dependence of the fractional resonance frequency. The solid lines are fits using Eq. (1), using MattisBardeen. The NbTiN on $\mathrm{SiO}_{x}$ data follow a superposition of a logarithmically temperature dependent permittivity (dashed line) and Mattis-Bardeen [Eq. (1)]. We choose $T_{0}=350 \mathrm{mK}$.

of Si $\left(T_{c}=15.5 \mathrm{~K}, \rho=84 \mu \Omega \mathrm{cm}\right.$, and $\left.R R R=1.0\right)$. We have also removed the exposed substrate surface from the region with large electric fields: fully straight, $50 \mathrm{~nm}$ thick, NbTiN resonators are made on $\mathrm{Si}$, aligned along the $\langle 110\rangle$ axis of the Si substrate $\left(T_{c}=13.6 \mathrm{~K}, \rho=142 \mu \Omega \mathrm{cm}\right.$, and $\left.R R R=0.96\right)$. Using $\mathrm{KOH}$ wet etching, $0.9 \mu \mathrm{m}$ deep grooves [dashed lines in Fig. 1(a)] are etched in the gaps along the full length of the resonators. As a reference and to clarify the influence of the metal edges, a straight, $50 \mathrm{~nm}$ thick NbTiN resonator is made where the Si substrate is not removed.

The frequency noise is measured using a homodyne detection scheme based on quadrature mixing. ${ }^{1,7,13}$ The samples are cooled to a temperature of $310 \mathrm{mK}$ using a He-3 sorption cooler placed in a $4.2 \mathrm{~K}$ liquid $\mathrm{He}$ cryostat. The sample stage is magnetically shielded with a superconducting shield. We use a low noise high electron mobility transistor amplifier with a noise temperature of $4 \mathrm{~K}^{14}$

The temperature dependence of the resonance frequency is shown in Fig. 3. For the NbTiN on $\mathrm{SiO}_{x}$ resonator we find a clear nonmonotonicity. The superposition [Eq. (1)] of the complex conductivity and logarithmically temperature dependent permittivity describes the data. The logarithmic dependence is consistent with resonant interaction of TLS with the electric fields at $k T>h f::^{10} \delta \epsilon / \epsilon=-\ln \left(T / T_{0}\right) 2 N p^{2} / \epsilon$ with $N$ the TLS density of states, $p$ the dipole moment, and $T_{0}$ an arbitrary reference temperature. The temperature dependence of the other resonators follows Mattis-Bardeen ${ }^{15}$ provided a broadening parameter ${ }^{16}$ of $\Gamma=15-20 \mu \mathrm{eV}$ is included in the density of states. ${ }^{17}$

The frequency noise spectra are shown in Fig. 4. The inset shows the dependence on the internal resonator power $P_{\text {int }}$. The noise spectra follow $S_{f_{0}} / f_{0}^{2} \propto 1 / f^{0.3-0.6}$, until a rolloff frequency on the order of $10 \mathrm{kHz}$. This roll-off arises from the resonator-specific response time, set by the loaded quality factor and resonator frequency. The NbTiN on $\mathrm{SiO}_{x}$ resonator has the highest frequency noise, at $P_{\text {int }}=-30 \mathrm{dBm}: S_{f_{0}} / f_{0}^{2}(1 \mathrm{kHz})=-195 \mathrm{dBc} / \mathrm{Hz}$. This is $3 \mathrm{~dB}$ noisier than the standard, $300 \mathrm{~nm}$ thick, NbTiN resonator which has a noise level of $-198 \mathrm{dBc} / \mathrm{Hz}$. The noise remains at $-198 \mathrm{dBc} / \mathrm{Hz}$ when decreasing the metal thickness by a factor of $6(8 \mathrm{~dB})$, from 300 to $50 \mathrm{~nm}$. Clearly, the noise is decreased with $7 \mathrm{~dB}$ for the resonator with grooves in the 


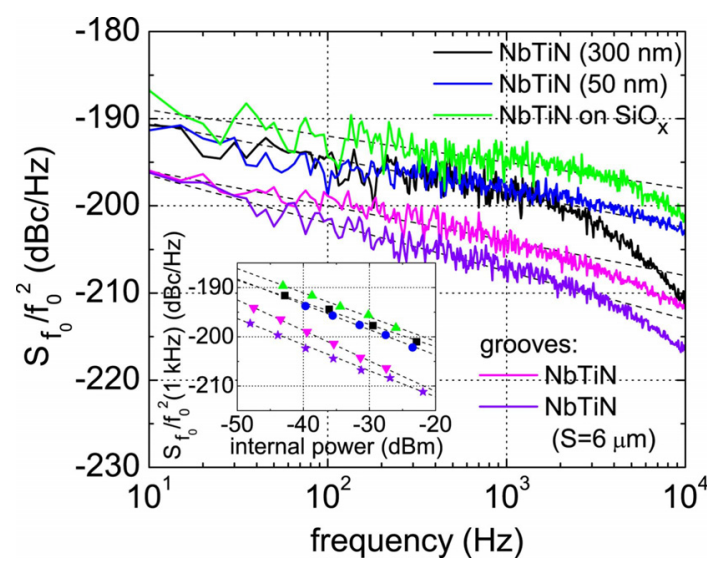

FIG. 4. (Color online) The normalized frequency noise spectra of NbTiN, NbTiN on $\mathrm{SiO}_{x}$, and NbTiN with grooves etched in the gaps. The widths are $S=3 \mu \mathrm{m}$ and $W=2 \mu \mathrm{m}$ except for the wide resonator with grooves: $S=6 \mu \mathrm{m}$ and $W=2$. The bath temperature is $310 \mathrm{mK}$ and the internal resonator power is $P_{\text {int }} \approx-30 \mathrm{dBm}$. The inset shows the power dependence, see Fig. 3 for the legend. Dashed lines are fits to the spectral shape and power dependence.

gaps, having $S_{f_{0}} / f_{0}^{2}(1 \mathrm{kHz})=-205 \mathrm{dBc} / \mathrm{Hz}$. Moreover, the noise is reduced over the whole range of spectral frequency and internal resonator power. For the wider resonator with $S=6 \mu \mathrm{m}$ the noise is $2 \mathrm{~dB}$ lower at $-207 \mathrm{dBc} / \mathrm{Hz}$. We find that the frequency noise follows $S_{f_{0}} / f_{0}^{2} \propto 1 / P_{\text {int }}^{0.4-0.6}$, up to powers of $P_{\text {int }} \sim-25 \mathrm{dBm}$.

The data and analysis show that NbTiN is a clean material and point toward $\mathrm{SiO}_{x}$ as the dominant source of noise. First, the exposed $\mathrm{Si}$ surface dominates the noise as its removal decreases the noise considerably. Second, when placing $\mathrm{SiO}_{x}$ below or on top of NbTiN the noise increases (Fig. 4 and Ref. 7). Third, the analysis in Fig. 2 indicates that the NbTiN surface is clean compared to that of $\mathrm{Si}$, as the metal surfaces influence the noise more strongly than the exposed substrate. In addition, the monotonic temperature dependence of the resonance frequency down to $350 \mathrm{mK}$ indicates that $\mathrm{NbTiN}$ has a minimal dielectric layer, in contrast to $\mathrm{Nb}$, $\mathrm{Ta}$, and $\mathrm{Al}$ (Ref. 7) as well as $\mathrm{NbTiN}$ on $\mathrm{SiO}_{x}$. Moreover, the metal edges are not dominant, as the noise level is independent of the thickness of the metal. Finally, the removal of dielectrics from the gaps leads to a decrease in the capacitance $C$ in Eq. (2). Hence, if the metal surfaces dominate, the noise would increase. Quantitatively, we estimate $\kappa(1 \mathrm{kHz}) \approx 5 \times 10^{-27} 1 / \mathrm{Hz}$ for $\mathrm{SiO}_{x}$, assuming $t=3 \mathrm{~nm}$. Importantly, the noise reduction is significant: it is $7 \mathrm{~dB}$ below our standard NbTiN on Si resonators and $11 \mathrm{~dB}$ below the lowest values reported for coplanar waveguide resonators by Gao et al..$^{5}$ In addition, the noise is $2 \mathrm{~dB}$ lower when widening to $S=6 \mu \mathrm{m}$, which is consistent with our calculation $[1.9 \mathrm{~dB}$, see Fig. 2(b)] and shows that further improvements can be obtained by widening the resonator.

A particular approach to remove the substrate was followed in Ref. 18 using $\mathrm{Al}$ on Si resonators. By etching the substrate isotropically, the noise was reduced to a level of $S_{f_{0}} / f_{0}^{2}(1 \mathrm{kHz})=-189 \mathrm{dBc} / \mathrm{Hz}$ at maximum power. Due to the undercut the influence of the exposed substrate surface as well the substrate-metal interface on the noise could not be distinguished.

The data in Fig. 4 provide a clear guide to low noise superconducting resonators, by using $\mathrm{NbTiN}$ and removing the exposed substrate surface from the region with the largest electric fields. Importantly, we show that both the removal of dielectrics as well as the widening of the resonator leads to a significant decrease in the noise. Hence, both approaches can be considered to be additive to decreasing the noise. Our approach can be implemented for lumped element resonators $^{8,19}$ as well: by using a $\langle 100\rangle$-oriented Si wafer and aligning the fingers and edges of the interdigitated capacitor along the two perpendicular $\langle 110\rangle$ axes, grooves can be etched with a minimal amount of undercut. Interestingly, our resonators with grooves also have higher quality factors at high internal power levels as well as at the single microwave photon levels needed for circuit quantum electrodynamics. ${ }^{12}$

To conclude, we have reduced the frequency noise by using $\mathrm{NbTiN}$ and removing the substrate from the region with the highest electric fields. This indicates that the exposed $\mathrm{Si}$ substrate surface is the main source of the noise, hence the contribution to noise from the NbTiN surface is not dominant. The followed approach is a straightforward route to low frequency noise in superconducting resonators.

The authors thank J. M. Martinis for stimulating discussions. The work was supported by the Pieter Langerhuizen Lambertuszoon funds of the Royal Holland Society of Sciences and Humanities and by the EU NanoSciERA project "Nanofridge."

${ }^{1}$ P. K. Day, H. G. LeDuc, B. A. Mazin, A. Vayonakis, and J. Zmuidzinas, Nature (London) 425, 817 (2003).

${ }^{2}$ J. Schlaerth, A. Vayonakis, P. Day, J. Glenn, J. Gao, S. Golwala, S. Kumar, H. LeDuc, B. Mazin, J. Vaillancourt, and J. Zmuidzinas, J. Low Temp. Phys. 151, 684 (2008).

${ }^{3}$ A. Monfardini et al., "NIKA: A millimeter-wave kinetic inductance camera," Astron. Astrophys. (to be published).

${ }^{4}$ S. J. C. Yates, A. M. Baryshev, J. J. A. Baselmans, B. Klein, and R. Güsten, Appl. Phys. Lett. 95, 042504 (2009).

${ }^{5}$ J. Gao, J. Zmuidzinas, B. A. Mazin, H. G. LeDuc, and P. K. Day, Appl. Phys. Lett. 90, 102507 (2007).

${ }^{6}$ J. Gao, M. Daal, J. M. Martinis, A. Vayonakis, J. Zmuidzinas, B. Sadoulet, B. A. Mazin, P. K. Day, and H. G. LeDuc, Appl. Phys. Lett. 92, 212504 (2008).

${ }^{7}$ R. Barends, H. L. Hortensius, T. Zijlstra, J. J. A. Baselmans, S. J. C. Yates, J. R. Gao, and T. M. Klapwijk, Appl. Phys. Lett. 92, 223502 (2008).

${ }^{8}$ O. Noroozian, J. Gao, J. Zmuidzinas, H. G. LeDuc, and B. A. Mazin, AIP Conf. Proc. 1185, 148 (2009).

${ }^{9}$ R. Barends, H. L. Hortensius, T. Zijlstra, J. J. A. Baselmans, S. J. C. Yates, J. R. Gao, and T. M. Klapwijk, IEEE Trans. Appl. Supercond. 19, 936 (2009).

${ }^{10}$ W. A. Phillips, Rep. Prog. Phys. 50, 1657 (1987).

${ }^{11}$ J. Gao, M. Daal, A. Vayonakis, S. Kumar, J. Zmuidzinas, B. Sadoulet, B. A. Mazin, P. K. Day, and H. G. LeDuc, Appl. Phys. Lett. 92, 152505 (2008).

${ }^{12}$ R. Barends, N. Vercruyssen, A. Endo, P. J. de Visser, T. Zijlstra, T. M. Klapwijk, P. Diener, S. J. C. Yates, and J. J. A. Baselmans, Appl. Phys. Lett. 97, 023508 (2010).

${ }^{13}$ R. Barends, Ph.D. thesis, Delft University of Technology, 2009.

${ }^{14}$ N. Wadefalk, A. Mellberg, I. Angelov, M. E. Barsky, S. Bui, E. Choumas, R. W. Grundbacher, E. L. Kollberg, R. Lai, N. Rorsman, P. Starski, J. Stenarson, D. C. Streit, and H. Zirath, IEEE Trans. Microwave Theory Tech. 51, 1705 (2003).

${ }^{15}$ D. C. Mattis and J. Bardeen, Phys. Rev. 111, 412 (1958).

${ }^{16}$ R. C. Dynes, V. Narayanamurti, and J. P. Garno, Phys. Rev. Lett. 41, 1509 (1978).

${ }^{17}$ For $300 \mathrm{~nm}$ thick NbTiN, NbTiN on $\mathrm{SiO}_{x}$, and $50 \mathrm{~nm}$ thick NbTiN we find for $\alpha \beta / 4: 0.18,0.08$, and 0.33 , respectively. We infer a magnetic penetration depth $\lambda$ of 400, 240, and $270 \mathrm{~nm}$.

${ }^{18}$ B. A. Mazin, Ph.D. thesis, California Institute of Technology, 2004.

${ }^{19}$ S. Doyle, P. Mauskopf, J. Naylon, A. Porch, and C. Duncombe, J. Low Temp. Phys. 151, 530 (2008). 